\title{
A Theorem on Runge Problem for Analytic Curves (*) (**).
}

\author{
AnTONIO CASSA
}

Summary. - Runge problem for analytic curves means: given a curve $O$ of an open $\Omega$ in an analytic manifold $X$ is it possible to find a sequence of curves $\left\{C_{v}\right\}$ of $X$ which, restricted to $\Omega$, converge to $C$ in a suitable topology? My paper gives an affirmative answer when $X$ is $a$ Stein manifold of dimension three and $\Omega$ is a Runge and Stein open of $X$.

Runge problem for analytic curves means: given a curve $C$ of an open $\Omega$ in an analytic manifold $X$ is it possible to find a sequence of curves $\left\{C_{y}\right\}$ of $X$ which, restricted to $\Omega$, converge to $O$ in a suitable topology?

My paper gives an affirmative answer when $X$ is a Stein manifold of dimension three and $\Omega$ is a Runge and Stein open of $X$.

The essential idea of the proving procedure is to try to define $\theta$ as the zero set of two holomorphic functions $f_{1}, f_{2}$ of $\Omega$ which moreover generate in each point of $C$ the ideal sheaf associate to $C$.

In this case, infact two functions $f_{1}^{\prime}, f_{2}^{\prime}$ of $X$ "close " to $f_{1}, f_{2}$ define a curve $C^{\prime}$ of $X$ « close " to $C$.

It's known (see [2], Corollar $2 \mathrm{zu}$ Satz 9) that if $C$ is a regular curve, $C$ is ideally theoretically complete intersection; but there are examples of singular curves of $C^{3}$ for which this is not true (for instance: the reducible curve composed by the three coordinates axis in $C^{3}$ ).

However it's possible to prove (see Theorem 1) that under suitable hypothesis there exist two functions $f_{1}, f_{2}$ of $\Omega$ defining $C$ plus in case curves of $X$ through the singular points of $C$ and generating the ideal sheaf of $C$ in all its regular points.

Thus, taking two functions $f_{1}^{\prime}, f_{2}^{\prime}$ of $X$ close to $f_{1}, f_{2}$ and zero on the possible more curves we can find a curve $C^{\prime}$ of $X$ close to $O$ in $\Omega$.

I wish to thank the Professors P. GRIfFIths and H. Rossr for their help during my training.

\section{1. - Existence of many local birational projections.}

Let fix an hermitian metric on $C^{n}$.

(*) Entrata in Redazione 1'11 ottobre 1974 .

(**) This article reproduces the Ph. D. thesis of the author at Brandeis University in May 1974. 
Definitios 1. - Let $f$ be a holomorphic function in a neighborhood $U$ of $O$ in $C^{n}$ such that $f(0)=0$ but $f \neq 0$.

A linear subspace $L$ of $\boldsymbol{C}^{n}$ of dimension $n-1$ is said to be regular in respect to $f$ at $O$ if there exists a neighborhood $U^{\prime} \subset U$ of $O$ where $f$ is a finite function on the fibers of the projection $\pi_{L}: C^{n} \rightarrow L$ (that is $\left.\not \pi_{L}^{-1}(w) \cap f^{-1}(z) \cap U^{\prime}\right)<\infty$ for each $z \in \boldsymbol{C}$ and $w \in L$ ).

REMARK. - $L$ is regular with respect to $f$ in $O$ if and only if $f$ is regular in $z_{n}$ at $O$ for each coordinate system $\left(z_{1}, \ldots, z_{n}\right)$ in $C^{n}$ isometric with the fixed one and such that $L=\left\{z \in C^{n}: z_{n}=0\right\}$.

Definttion 2. - Let $V$ be an analytic subspace of pure dimension $k<n$ of a neighborhood $U$ of $O$ in $C^{n}$ containing $O$.

A linear subspace $L$ of $C^{n}$ is said to be regular in respect of $V$ at $O$ (and $\pi: C^{n} \rightarrow L$ regular) if there exists a neighborhood $U^{\prime} \subset U$ of $O$ such that:

1) $\pi\left(V \cap U^{\prime}\right)$ is an analytic subspace of pure dimension $k$ in $\pi\left(U^{\prime}\right)$;

2) $\pi^{-1}: \pi\left(V \cap U^{\prime}\right) \rightarrow V$ is meromorphic.

REMARK. - When the condition 1) is satisfied, the condition 2) is equivalent to claim that $\pi^{*}: \mathcal{M}_{\pi(V), 0} \rightarrow \mathcal{M}_{V 0}$ is an isomorphism; where $\mathcal{M}_{V, 0}$ is the stalk of germs of meromorphic functions of $V$ at $O$ (see [1]).

Proposition 1. - Let $V$ be the germ at $O$ of an analytic subspace of pure dimension $k \leqslant n-1$ in $C^{n}$.

Let $r$ be a positive integer such that $k+1 \leqslant r \leqslant n-1$, the family of all linear subspaces of $\boldsymbol{C}^{n}$ of dimension $r$ non regular in respect to $V$ at $O$ is aset of measure zero in $\operatorname{Gr}(r, n)$.

Proof. - Let's procede by induction from $n-1$ to $k+1$.

LEMma. - For each funetion $f$ holomorphic at $O$ in $C^{n}$ let $f_{h}$ be the first homogeneous polynomial not identically zero in the expansion of $f$ at $O$, and let $W=\left\{x \in \boldsymbol{P}^{n-1}: f_{h}^{*}(x)=0\right\}$.

To each point $x \in \boldsymbol{P}^{n-1}-W$ corresponds (by the natural duality between $\boldsymbol{P}^{n-1}$ and $\operatorname{Gr}(n-1, n))$ a linear subspace $L_{x}$ of $\boldsymbol{C}^{n}$ of dimension $n-1$ regular in respect to $f$ at $O$.

Thus the set of all the linear subspaces non regular in respect to $f$ at $O$ is a set of measure zero in $\operatorname{Gr}(n-1, n)$.

LEMMA's PROOF. - Since $x \in P^{n-1}-W$, the normal line $N$ to $L_{x}$ is not contained in the cone $\left\{x \in \boldsymbol{C}^{n}: f_{h}(x)=0\right\}$.

Let $a_{n}$ be a vector of $N$ of lenght 1 and let $a_{1}, \ldots, a_{n-1}$ be an orthonormal base of $L_{x}$ and $l: C_{\zeta}^{n} \rightarrow C_{x}^{n}$ the isometry sending the base $\left(a_{1}, \ldots, a_{n}\right)$ in the natural base of $C^{n}$. 
The new function $g=f \circ l$ has total order $h$ as $f$ and since $f_{h}\left(a_{n}\right) \neq 0$ then $g_{h}(0, \ldots, 0,1) \neq 0$, that is $g$ is regular in $z_{n}$ at $O$.

INDUCTION: $r=n-1$. Let $\mathfrak{f}$ be the ideal of $V$ in $\mathcal{O}_{0}($ stalk at $O$ in $\mathcal{O}), A=\mathfrak{O} / S$ and $p: \mathcal{O} \rightarrow \mathcal{A}$ the quotient map.

Choose $f \in T$ non identically zero, the subset $A$ of $P^{n-I}$ of linear subspaces $L^{\prime}$ of dimension $n-1$ regular in respect to $f$ at $O$ has as complement $W$ in $P^{n-1}$ an algebraic variety.

The map $\pi^{\prime}: V \rightarrow L^{\prime}$ is finite in a neighborhood of $O$ (Weierstrass preparation theorem applied to $f)$, so $V^{\prime}=\pi(V)$ is an analytic subspace of dimension $k$ in a neighborhood of $O$ in $L^{\prime}$ (proper mapping theorem).

Let $\mathfrak{S}^{\prime}$ be the ideal of $V^{\prime}$ in $\mathcal{O}_{0}^{\prime}, \mathcal{A}^{\prime}=\mathcal{O}_{0}^{\prime} / \mathfrak{T}^{\prime}$ and $p^{\prime}: \mathcal{O}_{0}^{\prime} \rightarrow \mathcal{A}^{\prime}$.

Because the map $\pi^{\prime *}: \mathfrak{A}^{\prime} \rightarrow \mathfrak{A}$ is injective we can consider $\mathfrak{A}^{\prime}$ a subspace of $\mathfrak{A}$.

Let write $\eta_{n}=p\left(z_{n}\right)$ we want to show that $\mathcal{A}=\mathscr{A}\left[\eta_{n}\right]$ and $\eta_{n}$ is algebraic on $\mathcal{A}^{\prime}$.

Since $L^{\prime}$ is regular in respect to $f$ at $O$, we can find a orthonormal base $\left(v_{1}, \ldots\right.$, $v_{n-1}, v_{n}$ ) of $\boldsymbol{C}^{n}$ (where $\left(v_{1}, \ldots, v_{n-1}\right)$ is an arbitrary orthonormal base of $L^{\prime}$ ) giving a coordinate system $\left(z_{1}, \ldots, z_{n}\right)$ in $\boldsymbol{C}^{n}$ for which $f$ is regular in $z_{n}$ at $O$.

Then $f=h Q$, where $h \in \mathcal{O}^{\prime}\left[z_{n}\right]$ is a Weyerstrass polynomial and $Q$ is a unity of $\mathcal{O}$, therefore $h \in \mathcal{F}$. Say

With $a_{i} \in \mathcal{O}^{r}$.

$$
h=z_{n}^{\alpha}+\sum_{i<\alpha} a_{i} z_{n}^{i}
$$

Then in $\mathcal{A}$ we have $0=\eta_{n}^{\alpha}+\sum p\left(a_{i}\right) \eta_{n}^{i}$, that is $\eta_{n}$ algebraic on $\mathfrak{A}^{\prime}$.

Let $a \in \mathcal{A}, a=p(g)$ with $g \in \mathcal{O}$, by the division algorithm $g=h H+\sum b_{i} z_{n}^{i}$ with $b_{i} \in \mathcal{O}^{\prime}$; in $\mathcal{A}$ this means $a=\sum p\left(b_{i}\right) \eta_{n}^{i}$ that is $a \in \mathcal{O}^{\prime}\left[\eta_{n}\right]$.

Proceeding analogously we can find $f^{\prime} \in \mathfrak{T}^{\prime}, f^{\prime} \neq 0$ and $L^{\prime \prime}$ subspace of dimension $n-2$ of $L^{\prime}$ regular with $r^{2}$ espect to $f^{\prime}$ (almost all $L^{\prime \prime}$ are good).

Let write $V^{\prime \prime}=\pi^{\prime \prime}\left(V^{\prime}\right)$, this is a subspace of dimension $k^{\prime}$ in $L^{\prime \prime}$.

Let define $\mathfrak{S}^{\prime \prime}, \mathfrak{A}^{\prime \prime}, p^{\prime \prime}, \pi^{\prime \prime *}$, as above.

Choose $v_{1}, \ldots, v_{n-2}$ in $L^{\prime \prime}$ and define $\eta_{n-1}$, we can show again $\mathcal{A}^{\prime}=\mathcal{A}^{\prime \prime}\left[\eta_{n-1}\right]$ with $\eta_{n-1}$ algebraic on $\mathcal{A}^{\prime \prime}$; therefore $\mathcal{A}=\mathcal{A}^{\prime \prime}\left[\eta_{n-1}, \eta_{n}\right]$.

Let $F^{\prime}$ denote the quotient field of $\mathcal{A}$, $\mathscr{F}^{\prime}$ the quotient field of $\mathcal{A}^{\prime}, F^{\prime \prime}$ the quotient field of $\mathfrak{H}^{\prime \prime}$.

Then $\mathfrak{F}^{\prime}=\mathfrak{F}^{\prime \prime}\left(\eta_{n-1}, \eta_{n}\right)$.

Following the primitive element theorem (Van der Werden) is possible to check that for all the couples $\left(c_{n-1}, c_{n}\right) \in C^{2}$ such that $\left|c_{n-1} v_{n-1}+c_{n} v_{n}\right|=1$ except a finite set holds

$$
\mathscr{F}=\mathscr{F}^{\prime \prime}\left(c_{n-1} \eta_{n-1}+c_{n} \eta_{n}\right)
$$

Let choose $v_{n-1}^{\prime}=c_{n-1} v_{n-1}+c_{n} v_{n}$ such that the linear subspace $\tilde{L}^{\prime}$ generated by $v_{1}, \ldots, v_{n-2}, v_{n-1}$ is regular in respect to $f$ yet.

This is possible because $L^{\prime \prime}$ is represented in $\boldsymbol{P}_{n-1}$ by a projective line containing the point representing $L^{\prime}$. 
Since $L^{\prime} \notin W, L^{\prime \prime}$ meets $W$ in a finite set.

Choosing $\tilde{L}^{\prime}$ in $L^{\prime \prime}$ out of this set and out of the set of the $\tilde{L}^{\prime}$ where the primitive element in the form stated above does not hold, for all these $\tilde{L}^{\prime}$ we have $\mathscr{F}=\widetilde{F}$.

So $\tilde{\pi}^{\prime-1}: \tilde{V}^{\prime} \rightarrow V$ is meromorphic.

We want to show that such $\tilde{L}^{\prime}$ are represented by almost all the points of $\boldsymbol{P}^{n-1}$.

We arrived to $\tilde{L}^{\prime}$ choosing $L^{\prime}$ out of $W$, choosing a projective line $L^{\prime \prime}$ in $\boldsymbol{P}^{n-1}$ containing $L^{\prime \prime}$ and regular in respect to $f$, almost all the $L^{\prime \prime}$ have this property; at last choosing $\tilde{L}^{\prime}$ on $L^{\prime \prime}$ out of a finite set.

By the Tonelli Theorem it follows that the set $C$ of all the points of $\boldsymbol{P}^{n-1}$ non regular in respect to $V$ has measure zero, since almost all the projective lines trough $L^{\prime}$ meet $C$ in a set of measure zero.

INDUCTION. - Let's suppose we showed the theorem for each $r$ in the interval $r_{0}+1, \ldots, n-1$ (with $r_{0} \geqslant r+1$ ) and let's show it for $r=r_{0}$.

Let $L^{\prime}$ be one of the spaces of dimension $r_{0}+1$ regular in respect to $V$ and let $\pi: V \rightarrow L^{\prime}$ be the natural projection.

Let's consider $V^{\prime}=\pi^{\prime}(V)$ in a neighborhood of $O$ in $L^{\prime}$.

If we apply the induction with ambient space $L^{\prime}$ instead of $C^{n}$ and the analytic subspace $V^{\prime}$ instead of $\nabla$, we can claim that almost all the linear subspaces $L^{n}$ of dimension $r_{0}$ of $L^{\prime}$ are regular in respect to $V^{\prime}$ and hence to $V$.

Therefore almost all the points $L^{\prime}$ in $\operatorname{Gr}\left(r_{0}+1, n\right)$ are regular in respect to $V$ and almost all the linear subspaces of dimension 1 of $\operatorname{Gr}\left(r_{0}+1, n\right)$ passing trough $L$ are regular in respect to $V$.

It is possible to find an open of $\operatorname{Gr}\left(r_{0}+1, n\right)$ isomorphic to $\boldsymbol{C}^{\left(r_{\mathrm{s}}+1\right)\left(n-r_{0}-1\right)}=\boldsymbol{C}^{s}$ such that the linear subspaces of dimension 1 in $\operatorname{Gr}\left(r_{0}+1, n\right)$ which don't come from lines of $C^{s}$ make a set of measure zero in $\operatorname{Gr}\left(r_{0}, n\right)$.

We can also suppose that $C^{s-1}=\left\{z \in C^{s}: z_{s}=0\right\}$ meets the set of all the non regular points of $\boldsymbol{C}^{s}$ in a set of measure zero.

Almost all the lines of $C^{s}$ meet $C^{s-1}$ only in one point, these lines then make a trivial bundle of base $C^{s-1}$ and fiber $\boldsymbol{P}^{s-1}$ (in the fiber over $z_{0} \in C^{s-1}$ there are the lines trough $z_{0}$ ).

Since almost all the points of almost all the fibers are regular and the bundle is almost all $\operatorname{Gr}\left(r_{0}, n\right)$ we can conclude that the set of all the points of $\operatorname{Gr}\left(r_{0}, n\right)$ non regular in respect to $V$ make a set of measure zero.

\section{2. - Existence of many distinguishing projections.}

From now on $A_{r}$ will indicate the $r$-Hausdorff measure in $C^{n}$.

Proposition 2. - Let $D$ be a subset of $C^{n}-\{0\}$ such that $\Lambda_{r}(D)=0$ for $r>2 d$. Let write

$$
\mathfrak{L}_{k}=\{L \in \operatorname{Gr}(k, n): L \cap D \neq \emptyset\} .
$$


Then

$$
A_{r}\left(\mathcal{L}_{k}\right)=0 \quad \text { for } r>2 d+2(k-1)(n-k)
$$

Therefore the Lebesgue measure in $\operatorname{Gr}(k, n) \Lambda_{2 k(n-k)}\left(\mathfrak{L}_{k}\right)=0$ for $d<n-k$.

Proof. - Let write $\mathcal{A}_{k}=\left\{(x, L) \in\left(C^{n}-\{0\}\right) \times \operatorname{Gr}(k, n): x \in L\right\}$,

$$
P_{1}: \mathcal{A}_{k} \rightarrow C^{n}-\{0\}, \quad P_{2}: \mathscr{A}_{k} \rightarrow \operatorname{Gr}(k, n)
$$

Since $\mathcal{L}_{k}=P_{2}\left(P_{1}^{-1}(D)\right)$ and $P_{2}$ is locally lipschitzian, it is enough to show that

$$
A_{r}\left(P_{1}^{-1}(D)\right)=0 \quad \text { for } r>2 d+2(k-1) \times(n-k) \text { in }\left(C^{n}-\{0\}\right) \times \operatorname{Gr}(k, n) .
$$

Let $x \in D, P_{1}^{-1}(x) \subset\{x\} \times \operatorname{Gr}(k, n)$ is equivalent, by isometries in $\operatorname{Gr}(k, n)$ to Gr $(k-1, n-1)$ and thus:

$$
A_{r}\left(P_{1}^{-1}(x)\right)=0 \quad \text { for } r>2(k-1) \times(n-k)
$$

Let $a, b$ positive real numbers, $P_{1}^{-1}(D)$ is a measurable subset of $\mathcal{A}_{k}$ in respect to the measure

$$
\Lambda_{2 d+a} \times \Lambda_{2(k-1)(n-k)+b}=\Lambda_{2 d+2(k-1)(n-k)+a+b}
$$

and, using the Tonelli's Theorem, is possible to show that has measure zero.

CoRollary 1. - Let $V$ be an analytic subspace of an open $\Omega$ in $C^{n}$.

Let's suppose $\Lambda_{r}(\bar{V})=0$ for each $r>2 k$, and let's fix $x_{0} \in V$, almost all the affine linear subspace $L$ of dimension $n-k-1$ trough $x_{0}$ have the properties:

1) $\pi^{-1}\left(\pi\left(x_{0}\right)\right) \cap \bar{V}=\left\{x_{0}\right\}$

2) there exists a neighborhood $U$ of $x_{0}$ such that

$$
\pi^{-1}(\pi(U)) \cap \bar{V}=U \cap V
$$

Remark. - Let $X$ be a submanifold of dimension $n$ in $C^{N}$. Almost all the linear subspaces $L \in \operatorname{Gr}(n, N)$ have the property that there exists a neighborhood $U$ of $x_{0}$ in $X$ where the natural projection $\pi_{L}: U \rightarrow L$ gives local coordinates.

Proof. - Let $L \in \mathrm{Gr}(n, N)$, $\pi_{L}$ does not give local coordinates at $x_{0}$ in $X$ if and only if $\operatorname{rank} J_{x}\left(\left.\pi\right|_{X}\right)\left(x_{0}\right)<n$, that is if and only if $\operatorname{dim}\left[L \cap\left(T_{x_{0}}(X)\right)^{\perp}\right] \geqslant 1$; such linear subspaces $L$ make an analytic subspace of $\operatorname{Gr}(n, N)$. 
Proposimion 3. - Let $\Omega$ be an open $C^{n}, C$ a curve of $\Omega$ such that $\Lambda_{r}(\bar{C})=0$ for each $r>2$.

For almost all the linear subspaces $L \in \operatorname{Gr}(2, n)$ the projection $\pi: C^{n} \rightarrow L$ has the following properties:

1) for each $x_{i} \in \operatorname{sing}(C)$ there exists a neighborhood $U_{i}$ of $x_{i}$ such that $\pi\left(U_{i} \cap C\right)$ is a curve in $\pi\left(U_{i}\right)$;

2) $\pi^{-1}: \pi\left(U_{i} \cap C\right) \rightarrow C$ is meromorphic;

3) $\pi^{-1}\left(\pi\left(U_{i}\right)\right) \cap \bar{O}=U_{i} \cap C$.

PRoof. - It follows from Propositions 1 and 2 and the fact that sing $(C)$ is countable.

\section{3. - Local complete intersection.}

Propositron 4. - Let $\Omega$ be an open of $C^{n}, C$ a curve of $\Omega$ such that $A_{r}(\bar{C})=0$ for each $r>2$.

For each $x_{i} \in \operatorname{sing}(C)$ there exists an affine linear space $L_{i}$ of $C^{n}$ such that:

1) $L_{i} \cap C=\left\{x_{i}\right\}$;

2) $L_{i} \cap L_{j}=\emptyset$ if $i \neq j$;

and for each $x_{i} \in$ sing $C$ there exists an open neighborhood $A_{i}=U_{i}^{\prime} \times C^{n-2}$ of $L_{i}$ and holomorphic functions on $A_{i}, h_{i 1}, \ldots, h_{i n-1}$ such that:

3) $\left\{x \in A_{i}: h_{i j}(x)=0\right.$ for each $\left.j=1, \ldots, n-1\right\}=\left(C \cap A_{i}\right) \cup L_{i}$;

4) $h_{i 1 x}, \ldots, h_{i, n-1, \infty}$ generate $\tilde{\gamma}_{e, x}$ for each $x \in O \cap A_{i}-\left\{x_{i}\right\}$.

Proof. - Let's consider $L \in \operatorname{Gr}(2, n)$ and $\pi: C^{n} \rightarrow L$ as in Prop. 3 and let's put on $\boldsymbol{C}^{n}$ a coordinate system such that

$$
L=\left\{x \in \boldsymbol{C}^{n}: z_{j}(x)=0 \text { for } j=3, \ldots, n\right\} .
$$

Let's write $U_{i}^{\prime}=\pi\left(U_{i}\right), C_{i}=U_{i} \cap C, C_{i}^{\prime}=\pi\left(C_{i}\right)$.

Since $\pi^{-1}: C_{i}^{\prime} \rightarrow C_{i}$ is meromorphic there exist $m_{i 2}, \ldots, m_{i n-1}$ meromorphic on $C_{i}^{\prime}$ such that:

$$
\pi^{-1}(x)=\left(z_{1}(x), z_{2}(x), m_{i 2}(x), \ldots, m_{i, n-1}(x)\right) \quad \text { for each } x \in C_{i}^{\prime}
$$

Rechoosing the $U_{i}^{\prime}$ we can find functions $f_{i}, f_{i 2}, \ldots, f_{i n-1}, g_{i 2}, \ldots, g_{i, n-1}$ holomorphic on $U_{i}$ such that

$$
m_{i j}(x)=f_{i j}(x) / g_{i j}(x) \quad \text { for each } x \in C_{i}^{\prime}, x_{i} \in \operatorname{sing} C, j=2, \ldots, n-1,
$$


$\left\{x \in U_{i}^{\prime}: f_{i}(x)=0\right\}=O_{i}^{\prime}$ and furthermore $f_{i x}$ generates $\gamma_{\sigma_{i, x}^{\prime}}$ for each $x \in U_{i}^{\prime}$, and besides each $g_{i j}(x)=0$ at most in $\pi\left(x_{i}\right)$ on $C_{i}^{\prime}$.

Let's define the functions $h_{i j}$ holomorphic on $A_{i}=\pi^{-1}\left(U_{i}^{\prime}\right)$ in this way:

$$
h_{i 1}(x)=f_{i}(\pi(x)), \quad h_{i j}(x)=g_{i j}(\pi(x)) z_{j_{+1}}(x)-f_{i j}(\pi(x)) \quad \text { for } j=2, \ldots, n-1
$$

Therefore:

$$
\left\{x \in A_{i}: h_{i j}(x)=0 \text { for } j=1, \ldots, n-1\right\}=C_{i} \cup L_{i}
$$

where $L_{i}=\left\{x \in C^{n}: z_{j}(x)=z_{j}\left(x_{i}\right)\right.$ for each $j=1,2$ or such that $\left.g_{i, j-1}\left(\pi\left(x_{i}\right)\right)=0\right\}$.

Moreover $h_{i, 1 x}, \ldots, h_{i, n \rightarrow 1, x}$ generate $\tilde{\gamma}_{c, x}$ for each $w \in A_{i} \cap O-\left\{x_{i}\right\}$ infact: let's indicate with $p_{i}: U_{i}^{\prime} \rightarrow C^{n}$ the meromorphic map

$$
p_{i}\left(z_{1}, z_{2}\right)=\left(z_{1}, z_{2}, \frac{f_{i 2}\left(z_{1}, z_{2}\right)}{g_{i 2}\left(z_{1}, z_{2}\right)}, \ldots, \frac{f_{i, n-1}\left(z_{1}, z_{2}\right)}{g_{i, n-1}\left(z_{1}, z_{2}\right)}\right)
$$

For each $x \in C_{i} \cap A_{i}-\left\{x_{i}\right\}$ there exists a neighborhood $U_{x}$ contained in $D_{i}-\left\{x_{i}\right\}$ such that $p_{i}$ is holomorphic on $D_{x}^{\prime}=\pi\left(U_{x}\right)$.

Let's write $C_{x}=C_{i} \cap U_{x}, C_{x}^{\prime}=O_{i}^{\prime} \cap U_{x}^{\prime}$.

Let $q$ holomorphic on $U_{x}$ and vanishing on $O_{x}$, then $q(p(y))=0$ for each $y \in C_{x}^{\prime}$ and then there exists a holomorphic function $b$ on $V_{x}^{\prime}$ such that $q(p(y))=f_{i}(y) b(y)$ for each $y \in U_{x}^{\prime}$ (that is $q(p(\pi(u)))=h_{i 1}(u) b(\pi(u))$ for each $u \in U_{x}$.)

The function $q(u)-q(p(\pi(u)))$ vanishes in $p\left(U_{x}^{\prime}\right)$ in ${V_{x}^{\prime}}^{\prime} \times C^{n-2}$.

The rest of the proof follows from this lemma:

Lemma. - Let $U$ be an open of $\boldsymbol{C}^{a} C \boldsymbol{C}^{n}$ and $\varphi: U \rightarrow \boldsymbol{C}^{n}$ holomorphic so defined

$$
\varphi\left(z_{1}, \ldots, z_{d}\right)=\left(z_{1}, \ldots, z_{d}, \varphi_{a+1}(z), \quad, \varphi_{n}(z)\right)
$$

Let's call $S=\varphi(U)$ and let $q$ be an holomorphic function on a neighborhood of $S$ and vanising on $S$.

Then for each $z \in S$ there exists a neighborhood $U_{z}$ of $z$ and functions $a_{a_{+1}}, \ldots, a_{n}$ holomorphic on $U_{z}$ such that

$$
q(y)=\left(z_{d+1}(y)-\varphi_{d+1}(y)\right) a_{d+1}(y)+\ldots+\left(z_{n}(y)-\varphi_{n}(y)\right) a_{n}(y)
$$

for each $y \in U_{z}$.

Proof of the lemma, - The map $\psi: U \times \boldsymbol{C}^{n-a} \rightarrow U \times \boldsymbol{C}^{n-d}$ defined by

$$
\psi\left(z_{1}, \ldots, z_{n}\right)=\left(z_{1}, \ldots, z_{d}, z_{d+1}-\varphi_{d+1}(z), \ldots, z_{n}-\varphi_{n}(z)\right)
$$

gives locally a change of coordinates in $U \times C^{n-d}$.

Moreover $\psi(S)=U \times\{0\}$ and $\psi \circ \phi=1_{U}$. 
Let's write $\left(\psi^{-1}\right)_{z}$ the inverse map of $\psi$ from a neighborhood of $\psi(z)$ to a neighborhood of $z$ and $q^{\prime}=q \circ\left(\psi^{-1}\right)_{z}, q^{\prime}$ is defined in a neighborhood of $\psi(z) \in U \times\{0\}$ and vanishing on $U \times\{0\}$, therefore there exist functions $b_{d+1}, \ldots, b_{n}$ holomorphic in a neighborhood of $\psi(z)$ such that

$$
q^{\prime}\left(w_{1}, \ldots, w_{n}\right)=w_{d+1} b_{d+1}(w)+\ldots+w_{n} b_{n}(w)
$$

therefore

$$
q\left(z_{1}, \ldots, z_{n}\right)=\left(z_{d+1}-\varphi_{a+1}(z)\right) b_{d+1}(\psi(z))+\ldots+\left(z_{n}-\varphi_{n}(z)\right) \cdot b_{n}(\psi(z))
$$

in a neighborhood of $z$.

Contrnuation of the proposition's proof. - Thus by the lemma

$$
q(u)-q(p(\pi(u)))=\left(z_{3}(u)-\frac{f_{i, 2}(u)}{g_{i, 2}(u)}\right) a_{2}(u)+\ldots+\left(z_{n}(u)-\frac{f_{i n-1}(u)}{g_{i n-1}(u)}\right) a_{n-1}(u)
$$

in a neighborhood of $x$.

Therefore:

$$
q(u)=h_{i 1}(u) a(u)+h_{i 2}(u) \frac{1}{g_{i 2}(u)} a_{2}(u)+\ldots+h_{i, n-1}(u) \frac{1}{g_{i, n-1}(u)} a_{n-1}(u) .
$$

Propositron 5. - Let $X$ be a Stein manifold of dimension $n, \Omega$ an open of $X$ and $C$ a curve of $\Omega$ with a finite set of singularities.

For each $x_{i} \in \operatorname{sing} C$ there exists an analytic subspace $Y_{i}$ of $X$ such that:

1) $\operatorname{dim}\left(Y_{i} \cap C\right)=\mathbf{0}, x_{i} \in Y_{i} \cap C$;

2) $Y_{i} \cap Y_{0}=\emptyset$ if $i \neq j$;

and an open neighborhood $A_{i}$ of $Y_{i}$ with $A_{i} \cap A_{j}=\emptyset$ if $i \neq j$ and holomorphic functions on $A_{i}, h_{i 1}, \ldots, h_{i, n-1}$ such that:

3) $\left\{x \in A_{i}: h_{i j}(x)=0\right.$ for each $\left.j=1, \ldots, n-1\right\}=\left(C \cap A_{i}\right) \cup Y_{i}$;

4) $h_{i 1, x}, \ldots, h_{i, n-1, x}$ generate $\tilde{\gamma}_{C, x}$ for each $x \in C \cap A_{i}-\left\{x_{i}\right\}$.

Proof. - Let's embed $X$ in $C^{N}$.

Applying the Prop. 2 and its Remark it's possible to find a $n$-dimensional linear subspace $L$ of $C^{N}$ and a neighborhood $U$ of sing $(C)$ in $X$ such that $\pi_{L}$ is holomorphic and does not send a component of $C$ to a point.

Applying the Prop. 4 to the space $L$, the open $\pi(U)$ and the curve $\pi(C \cap U)$ and pulling back $L_{i}, A_{i}, h_{i j}$ to $X$ we get the new $Y_{i}, A_{i}, h_{i j}$ as wished.

\section{4. - Complete intersection on compacts.}

From now on we'll have a continuous needing of the results contained in [2]. The following is a brief exposition of some of them. 
Let $\mathcal{F}$ be an analytic sheaf on a Stein manifold $X$.

Let's suppose there exist $f_{1}, \ldots, f_{r} \in \Gamma(X, F)$ generating $\mathfrak{F}_{x}$ for each $x \in X$.

At a point $x \in X$ the least number of generators of $\mathscr{F}_{x}$ is given by the dimension on $\boldsymbol{C}$ of the vector space $L_{x}(\mathscr{F})=\mathscr{F}_{x} / \mathcal{M}_{x} \cdot \mathscr{F}_{x}$.

Globally the existence, for a fixed integer $s$, of $s$ global sections of $\mathscr{F}: g_{1}, \ldots, g_{s}$ generating $\mathfrak{F}$ in each point is equivalent to the existence of a global holomorphic section for a kind of bundle $E(\mathscr{F}, f, s) \rightarrow X$ called Endromisbundel.

The Endromisbundel is an open of $X \times C_{r s}$ obtained subtracting analytic spaces defined by $f_{1}, \ldots, f_{r}$ and by the integers $\left\{\operatorname{dim} L_{x}(\mathfrak{F})\right\}_{x \in X}$.

Let's define for each positive integer $k$ :

$$
\bar{Y}_{k i}=\left\{x \in X: \operatorname{dim} L_{x}(\mathcal{F}) \geqslant k\right\}
$$

the $Y_{k}$ make a decreasing sequence of analy tic subspaces of $X$, empty from $r+1$; on $X_{x}=Y_{k}-Y_{k_{+1}}$ the Endromisbundel is an holomorphic fiber bundle with fiber the topological space of matrices $F_{r, s, k}$ homotopic to the space $W_{s k}$ of the unitary hortonormal $k$-frames of $\boldsymbol{C}^{s}$.

An important result of Forster and RAMsPotT (Satz 5 and 6 of [2]) claims that if $X$ is Stein the existence of holomorphic sections for the Endromisbundel is equivalent to the existence of continuous sections.

The problem of the least number of generators for the sheaf in each point of $X$, is then reduced to a purely topological problem with main ingredients the spaces $X_{k}$ and the fibers $W_{s k}$.

Theorem 1. - Let $X$ be a Stein manifold of dimension $n \geqslant 3, \Omega$ a Stein open of $X$ and $C$ a curve of $\Omega$.

Let $\Omega^{\prime}$ be a Stein open relatively compact in $\Omega$ and let's write $C^{\prime}=C \cap \Omega^{\prime}$.

For each $x_{i} \in \operatorname{sing}\left(C^{\prime}\right)=\left\{x_{1}, \ldots, x_{s}\right\}$ there exists an analytic subspace $Y_{i}$ of $X$ such that:

1) $\operatorname{dim}\left(\bar{Y}_{i} \cap O\right)=0, x_{i} \in \bar{Y}_{i} \cap C$;

2) $Y_{i} \cap Y_{j}=\emptyset$ if $i \neq j$;

and there exists functions $h_{1}, \ldots, h_{n-1}$ holomorphic on $\Omega^{\prime}$ such that:

3) $\left\{x \in \Omega^{\prime}: h_{i}(x)=0\right.$ for $\left.j=1, \ldots, n-1\right\}=C^{\prime} \cup \bigcup_{n-1}^{s}\left(Y_{i} \cap \Omega^{\prime}\right)$;

4) $h_{1 x}, \ldots, h_{n-1, x}$ generate $\tilde{\delta}_{C^{\prime}, x}$ for each $x \in \operatorname{reg}\left(O^{\prime}\right)$.

Proof. - Let's consider $Y_{i}, A_{i}, h_{i j}$ as in Prop. 5.

Let's define:

the image

$$
\Phi_{i}: \mathcal{O}_{\mid A_{i}}^{n-1} \rightarrow \mathcal{O}_{\mid A_{i}} \quad \text { by } \Phi_{i}\left(f_{1}, \ldots, f_{n-1}\right)=\sum_{j=1}^{n-1} h_{i j} f_{j}
$$

$$
\gamma_{i}=\Phi_{i}\left(\mathcal{O}_{i A_{i}}^{n-1}\right)
$$

12 - Annali a Matematica 
is coherent subsheaf of $\mathcal{O}_{\left[A_{i}\right.}$ and

$$
y_{i, x}= \begin{cases}\mathcal{O}_{x} & \text { if } x \in A_{i}-\left(C \cup Y_{i}\right), \\ \mathfrak{J}_{C, x} & \text { if } x \in C \cap A_{i}-\left\{x_{i}\right\} .\end{cases}
$$

Let's define a sheaf $g$ in $\mathcal{O}_{\Omega}$ in this way: $\tilde{\gamma}_{x}=\tilde{\gamma}_{i, x}$ if $x \in A_{i}$ and $\tilde{\gamma}_{x}=\tilde{\gamma}_{c, x}$ if $x \in \Omega-\bigcup_{i=1}^{s} Y_{i}$.

The sheaf $\tilde{g}$ is coherent and since $\bar{\Omega}^{\prime}$ is compact in $\Omega$ Stein, there exists a chain of syzygies of $\gamma$ in $\Omega^{\prime}$ :

$$
\ldots \rightarrow \mathcal{O}_{\mid \Omega}^{r_{1}} \rightarrow \mathcal{O}_{\mid \Omega^{\prime}}^{r} \stackrel{f}{\rightarrow} \tilde{g}_{\mid \Omega^{\prime}} \rightarrow O
$$

The components of $f=\left(f_{1}, \ldots, f_{r}\right)$ therefore generate $\gamma_{x}$ in each point of $\Omega^{\prime}$.

Since we are interessed in finding $h_{1}, \ldots, h_{n-1} \in \Gamma\left(\Omega^{\prime}, \mathcal{O}\right)$ with the same property we have to show that there exists a continuous section of the Endromisbundel $E\left(\Omega^{\prime}, \mathcal{F}, f, n-1\right)$ on $\Omega^{\prime}$.

The functions $h_{i 1}, \ldots, h_{i n-1}$ generate $\gamma_{x}$ for each $x \in A$, so on $A_{i}^{\prime}=A_{i} \cap \Omega^{\prime}$ (Stein) $h_{i 1}\left|A_{i}^{r}, \ldots, h_{i n-1}\right| s_{i}^{z}$ define ([2], Hilfssatz 3 ) a continuous section of

$$
E\left(A_{i}^{\prime},\left.\mathscr{J}\right|_{A_{i}^{\prime}},\left.f\right|_{A_{i}^{\prime}}, n-1\right)=\left.E\left(\Omega^{\prime}, \mathscr{J}, f, n-1\right)\right|_{A_{i}^{\prime}} .
$$

Let $A^{\prime}=\bigcup_{i=1}^{s} A_{i}^{\prime}$.

Choose a triangulation on $\Omega^{\prime}$ which induces a triangulation on $C^{\prime}$ and on $\bar{Y}^{\prime}=$ $=\bigcup_{i=1}^{s}\left(Y_{i} \cap \Omega^{\prime}\right)$.

Refining the triangulation we can find a neighborhood $V$ of $Y^{\prime}$ which is a polyedron contained in $A^{\prime}$ and such that $V \cap C^{\prime}$ is contractible on $\left\{x_{1}, \ldots, x_{s}\right\}$.

To extend our continuous section from $V \cap C^{\prime}-Y^{\prime}$ to $O^{\prime}-Y^{\prime}$ it is enough (see [7], page 174):

$$
H^{\alpha+1}\left(C^{\prime}-Y^{\prime},\left(V \cap C^{\prime}\right)-Y^{\prime} ; \pi_{a}\left(W_{n-1, n-1}\right)\right)=0
$$

for each $q \geqslant 1$, because $\left.E(\mathcal{f}, f, n-1)\right|_{\sigma^{\prime}-Y^{\prime}}$ is a fiber bundle with fiber $F_{r, n-1, n-1}$ homotopic to $W_{n-1, n-1}$ (see [2], Satz 1, page 148).

Now

$$
H^{\alpha+1}\left(C^{\prime}-\bar{Y}^{\prime}, V \cap O^{\prime}-Y^{\prime}\right) \simeq H^{q+1}\left(C^{\prime}, V \cap C^{\prime}\right) \simeq H^{q+1}\left(C^{\prime}\right) \quad \text { for } q \geqslant 0
$$

by the excision first and by the contractibility of $V \cap C^{\prime}$ on $\left\{x_{1}, \ldots, x_{s}\right\}$ after; at last since $O^{\prime}$ is a curve $H^{q+1}\left(O^{\prime}\right)=0$ for each $q \geqslant 1$.

Let $W$ be a subpolyedron of $\Omega^{\prime}$ which is a neighborhood of $C^{\prime} \cup Y^{\prime}$ contractible on $C^{\prime} \cup T^{\prime}$, if $W$ is small enough the section of $\left.E(\gamma, f, n-1)\right|_{\sigma^{\prime} \cup Y^{\prime}}$ can be extended to $W$ (see [2], Hilfssatz 2, page 147 and proof of Satz 9, page 161). 
To extend the section from $W-\left(C^{\prime} \cup \bar{Y}^{\prime}\right)$ to $\Omega^{\prime}-\left(C^{\prime} \cup Y^{\prime}\right)$ is enough:

$$
H^{q+1}\left(\Omega^{\prime}-\left(C^{\prime} \cup Y^{\prime}\right), W-\left(C^{\prime} \cup Y^{\prime}\right), \pi_{q}\left(W_{n-1,1}\right)\right)=0 \quad \text { for each } q \geqslant 1,
$$

because $\left.E(\mathcal{J}, f, n-1)\right|_{\Omega^{\prime}-\left(C^{\prime} \cup Y^{\prime}\right)}$ is a fiber bundle with fiber $F_{r, n-1,1}$ homotopic to $W_{n-1,1}$.

Since $\pi_{q}\left(W_{n-1,1}\right)=0$ for $q \leqslant 2 n-4$ is sufficient to prove that

$$
H^{\alpha+1}\left(\Omega^{\prime}-\left(C^{\prime} \cup \bar{Y}^{\prime}\right), W-\left(C^{\prime} \cup Y^{\prime}\right)\right) \simeq H^{a+1}\left(\Omega^{\prime}, W\right) \simeq H^{a+1}\left(\Omega^{\prime}, C^{\prime} \cup \bar{Y}^{\prime}\right)
$$

vanishes for $q \geqslant 2 n-3$.

From the exact sequence

$$
\ldots \rightarrow H^{q}\left(C^{\prime} \cup Y^{\prime}\right) \rightarrow H^{q+1}\left(\Omega^{\prime}, C^{\prime} \cup Y^{\prime}\right) \rightarrow H^{q+1}\left(\Omega^{\prime}\right) \rightarrow \ldots
$$

since $H^{a}\left(C^{\prime} \cup Y^{\prime}\right) \simeq H^{b+1}\left(\Omega^{\prime}\right)=0$ for $q \geqslant n$ (see [6]), we can conclude

$$
H^{q+1}\left(\Omega^{\prime}, C^{\prime} \cup Y^{\prime}\right)=0 \quad \text { for } q \geqslant n .
$$

Therefore if $n \geqslant 3 H^{q+1}\left(\Omega^{\prime}, C^{\prime} \cup Y^{\prime}, \pi_{q}\left(W_{n-1,1}\right)\right)=0$ for each $q \geqslant 1$, thus there exists a holomorphic section of $E(\mathfrak{f}, f, n-1)$ on $\Omega^{\prime}$.

\section{5. - Convergence of analytic subsets.}

Proposition 6 . - Let $\Omega$ be an $n$-dimensional manifold.

Let $f, f_{v}: \Omega \rightarrow C^{n-d}$ holomorphic maps $(v \in N)$ with

$$
f=\lim _{v \rightarrow \infty} f_{v}
$$

Let's write $X=\{x \in \Omega: f(x)=0\}, X_{v}=\left\{x \in \Omega: f_{v}(x)=0\right\}$.

If $X$ has pure dimension $d$, then for each compact $K$ in $\Omega$ there exists an integer $v_{E}$ such that for $v \geqslant v_{R}: X_{v} \cap \frac{0}{K}$ has dimension $d$.

LEmma. - For each $x_{0} \in X$, there exists a neighborhood $U_{x_{0}}$ and an integer $v_{x_{0}}$ such that for $\nu \geqslant v_{x_{0}}: X_{\nu} \cap U_{x_{0}}$ has pure dimension $d$.

LEMma's PRoof. - We can change coordinates at $x_{0}$ such that $x_{0}=0$ and there exists a polycilinder $\Delta^{(n)}(0, r)$ where the projection $\pi: \Delta^{(n)}(0, r) \cap X \rightarrow \Delta^{(d)}(0, r)$ is a finite map.

We can also suppose that on $D=\left\{z \in C^{n}:\left(z_{1}, \ldots, z_{d}\right) \in \Delta^{(d)}(0, r)\right.$ and $\left|z_{i}\right|=r_{i}$ for $d+1 \leqslant i \leqslant n\}$ the lower bound of $|f|$ is a positive number $\delta$.

For $\nu \geqslant v_{x_{0}}$ big enough we have $\left\|f-f_{\nu}\right\|<\delta$ so that $\inf _{D}\left|f_{\nu}\right|>0$.

Then called $\Delta_{y}=\left(\{y\} \times C^{n-d}\right) \cap \Delta^{(n)}$ for each $y \in \Delta_{y}, X_{v} \cap \bar{\Delta}_{y}$ is all inside $\Delta_{y}$ 
and therefore is compact and finite, that is

$$
\operatorname{dim}\left(X_{p} \cap \Delta_{y}\right)=0 .
$$

Then $\operatorname{dim}\left(X_{\nu} \cap \Delta^{(n)}(0, r)\right) \geqslant d$ (see [3], Cor. 8, page 113).

But $\operatorname{dim}\left(X \cap \cap_{\nu} \Delta^{(n)}(0, r)\right) \leqslant d$, so $\operatorname{dim}\left(X_{\nu} \cap A^{(n)}(0, r)\right)=d$ for $v \geqslant v_{x_{0}}$.

From now on we will make a continuous referement to two papers ([4] and [5]) of King.

Most of the notations used here are taken from these papers.

As in KING [4] if $f$ is a holomorphic map from a manifold $\Omega$ to $C^{r}$ we denote:

$$
\begin{aligned}
& \eta(f)=\frac{1}{2 \pi i} \partial \log |f|^{2}, \\
& \omega(f)=d \eta(f)=\bar{\partial} \eta(f), \\
& R(f)=b\left[\eta(f) \wedge\left(\omega(f)^{n-k-1}\right)\right]-\left[(\omega(f))^{n-k}\right]=[X],
\end{aligned}
$$

if the zero set $X$ of $f$ is a pure $k$-dimensional subset of $\Omega$.

Proposition 7. - Let $\Omega$ be a $n$-dimensional manifold

$$
f_{\nu}: \Omega \rightarrow C^{r}(\nu \in \widetilde{N}=N \cup\{\infty\})
$$

holomorphic maps such that $f_{\infty}=\lim _{p \rightarrow \infty} f_{p}$.

Let's suppose the $X_{r}=\left\{x \in \Omega: f_{v}(X)=0\right\}$ are pure $(n-r)$ dimensional subsets of $\Omega$.

Then

$$
R\left(f_{\infty}\right)=\lim _{y \rightarrow \infty} R\left(f_{y}\right) \quad \text { in } \mathfrak{D}_{2(n-\eta)}(\Omega)
$$

Proof. - First of all we need the following:

LEMma 1. - For each $x_{0} \in X_{\infty}$ there exists a neighborhood $P_{x_{0}}$ and a costant $C_{x_{0}}$ such that:

$$
C_{x_{0}} \geqslant M\left(R\left(f_{v}\right)_{P_{x_{0}}}\right) \quad \text { for each } \nu \in N .
$$

where $M(T)$ is the mass of $T$ (see [5], page 192 and 201).

Proof. OF THE LEMMA. - Let's choose at $x_{0}$ a clear coordinate system for $X_{\infty}$ (see [5], page 201-202).

For each choose $I=\left(i_{1}, \ldots, i_{a}\right)$ with $d=n-r$ and $1 \leqslant i_{1} \leqslant \ldots \leqslant i_{d} \leqslant n$ we can find a polycilinder $P_{I}$ such that the map $\pi_{I}: P_{I} \cap X_{\infty} \rightarrow C^{d}$ is finite and $X \cap \partial_{I} P_{I}=\emptyset$ where $\partial_{I} P_{I}=\partial P_{I}-\left\{z \in C^{n}:\left|\tilde{i}_{i_{\alpha}}\right|=\delta_{i_{\alpha}}\right.$ for $\left.\alpha=1, \ldots, d.\right\}$

Then $\inf _{\partial_{I} P_{I}}\left|f_{\infty}\right|=\sigma_{I}>0$. 
Set $P_{I}^{\prime}=\pi_{I} P_{I}$ in $C^{d}$, for each $x \in P_{I}^{\prime}$ let write $L_{x}=\left\{z \in C^{n}: z_{i_{a}}=z_{i_{a}}(x)\right.$ for $\alpha=$ $=1, \ldots, d\}$, then $\#\left(L_{x} \cap X_{\infty} \cap P_{I}\right)=s_{I}\left(x, f_{\infty}\right)$ is finite and

$$
s_{I}\left(x, f_{\infty}\right)=\int_{L_{x} \cap \hat{\theta}_{I} P_{I}} \frac{\lambda_{I}\left(f_{\infty}\right)}{\left|f_{\infty}\right|{ }^{4(n-r)+2}}
$$

for the Bochner-Martinelli formula, where $\lambda_{I}\left(f_{\infty}\right)$ is a form which coefficients are polynomials in $f_{\infty}$ and its derivatives.

Thus $s_{I}\left(x, f_{\infty}\right)$ is an holomorphic function with positive integral values, then a constant $s_{I}\left(f_{\infty}\right)$, for $P_{I}$ is connected.

For $v$ big enough $\inf _{\partial_{I} P_{I}}\left|f_{\nu}-f_{\infty}\right|<\sigma_{\nu}$, so $s_{T}\left(f_{\nu}\right)$ is defined and for $v$ bigger than a suitable $y_{I}$ and we have $s_{I}\left(f_{v}\right)=s_{I}\left(f_{\infty}\right)$.

Let put $P_{x_{0}}=\bigcap_{I} P_{I}$; if $w$ is the usual Kahler form associated to an hermitian metric on $\Omega$ :

$$
\begin{aligned}
& \boldsymbol{M}\left(\left.R\left(f_{v}\right)\right|_{P_{x_{e}}}\right)=\int_{P_{x_{0}}} R\left(f_{y}\right)\left(w^{d}\right)=\int_{P_{x_{q}}}\left[-\left(\omega\left(f_{y}\right)^{n-d}\right)\right] \wedge w^{d} \leqq \\
& \leqq \sum_{I} \int_{P_{I}}\left[-\left(\omega\left(f_{p}\right)^{n-d}\right) \wedge\left(d z_{I} \wedge d \bar{z}_{I}\right)\right]=\sum_{I} \int_{P_{I}^{\prime}}\left(\int_{L_{x} \cap P_{I}}\left[-\left(\omega\left(f_{p}\right)\right)^{n-d}\right] d z_{I} \wedge d \bar{z}_{I}\right)= \\
& =\sum_{I} \int_{P_{I}^{\prime}}\left(\int_{L_{x} \cap \partial_{\partial_{I} P_{I}}}-\eta\left(f_{v}\right) \wedge \omega\left(f_{v}\right)^{n-\alpha-1}\right) d \bar{z}_{I} \wedge d \bar{z}_{I}= \\
& =\sum_{I} s_{I}\left(f_{v}\right) \operatorname{vol}\left(P_{I}^{\prime}\right)=\sum_{I} s_{I}\left(f_{\infty}\right) \operatorname{vol}\left(P_{I}^{\prime}\right)=O_{x_{a}}^{\prime} \text {. }
\end{aligned}
$$

For $C_{x_{0}}=\max \left\{C_{x_{0}}^{\prime}, M\left(\left.R\left(f_{1}\right)\right|_{P_{x_{0}}}\right), \ldots, M\left(\left.R\left(f_{y-1}\right)\right|_{P_{x_{0}}}\right)\right\}$ we have $M\left(\left.R\left(f_{v}\right)\right|_{P_{x_{0}}}\right) \leqslant C x_{0}$ for each $v \in N$.

Now we can easily prove the:

Lemad 2. - For each compact $K$ of $\Omega$ there exists a constant $C_{K}$ such that

$$
M\left(\left.R\left(f_{\nu}\right)\right|_{K}\right) \leqslant C_{K} \quad \text { for each } \nu \in N
$$

Proof of the Lemia 2. - The opens $P_{x}$ where $x \in X_{\infty} \cap K$ cover $X_{\infty} \cap K$, then there exists $x_{1}, \ldots, x_{m} \in X_{\infty} \cap K$ such that

$$
A=\bigcup_{a=1}^{m} P_{x_{a}} \supset X_{\infty} \cap K
$$

Then $\inf _{K \rightarrow A}\left|f_{\infty}\right|=\tau>0$ and for $\nu$ bigger than a suitable $\tilde{y}$, from $\sup \left|f_{v}-f_{\infty}\right|<\tau$, it follows $X_{v} \cap K \subset A$.

Thus

$$
M\left(\left.R(f)\right|_{K} ^{\circ}\right) \leqslant \sum_{\alpha=1}^{m} M\left(\left.R\left(f_{v}\right)\right|_{P x_{\alpha}}\right) \leqslant \sum_{*} C_{x_{a}}=C_{K}^{\prime} .
$$


For $C_{K}=\max \left\{C_{K}^{\prime}, M\left(\left.R\left(f_{1}\right)\right|_{K} ^{\circ}\right), \ldots, M\left(\left.R\left(f_{\tilde{\nu}-1}\right)\right|_{\hat{K}}\right)\right\}$ we have $M\left(\left.R\left(f_{v}\right)\right|_{k}\right) \leqslant C_{K}$ for each $\nu \in N$.

Continuation of the Proof of Proposimion 7. - Since the sequence $\left\{R\left(f_{v}\right)\right\}$ is bounded in mass on each compact and $b\left\{R\left(f_{y}\right)\right\}=\{0\}$, the sequence is bounded on $I_{2 k}^{\text {loc }}(\Omega)$ (see [5], page 196) and relatively compact.

Therefore there exists a subsequence $\left\{R\left(f y_{k}\right)\right\}$ converging to $T \in I_{2 k}^{\mathrm{loc}}(\Omega)$.

This current has support in $X_{\infty}$.

Infact let $x \notin X_{\infty}$ and $U_{x}$ a compact neighborhood of $x$ disjoint from $X_{\infty}$.

For $k$ bigger than a suitable $k^{\prime}$ we have $\sup _{V_{x}}\left|f_{v_{k}}-f_{\infty}\right|<\inf \left|f_{\infty}\right|$ and for each form $\varphi \in A_{2 k}^{0}\left(\stackrel{\circ}{U}_{x}\right) T(\varphi)=\lim _{k \rightarrow \infty} R\left(f_{v_{k}}\right)(\varphi)=\lim _{k \rightarrow \infty} 0=0$.

Therefore for [5], Proposition 3.1.3, page $200 T=\sum \gamma_{i}\left[X_{\infty, i}\right]$ where the $\gamma_{i}$ are positive integers and the $X_{\infty i}$, are the irriducible components of $X_{\infty}$.

From [4], page $47 R\left(f_{\infty}\right)=\sum c_{i}\left[X_{\infty, i}\right]$.

Let's consider $x_{i} \in \operatorname{reg}\left(X_{\infty, i}\right)$ and choose in a neighborhood $U$ of $x_{i}$ a coordinate system such that

$$
X_{\infty i} \cap U=C^{a} \cap U \text { and } z_{j}\left(x_{i}\right)=0 \quad \text { for } j=1, \ldots, n .
$$

Let's choose at 0 a polycilinder $P$ which meets $X_{\infty}$ only in $C^{d}$.

Let's call $L$ the ortogonal space to $C^{d}$ in 0

$$
\begin{aligned}
\gamma_{i}=M\left(\left.T\right|_{L \cap P}\right)=\left.T\right|_{L \cap P}(1)= & \lim _{k \rightarrow \infty} R\left(f v_{k}\right)(1)= \\
& =\lim _{k \rightarrow \infty} \int_{L \cap P}\left[-\left(\omega\left(f_{v_{k}}\right)\right)^{n-d}\right]=\lim _{k \rightarrow \infty} \int_{L \cap \partial P}\left[-\eta\left(f_{v_{k}}\right) \wedge \omega\left(f_{v_{k}}\right)^{n-d-1}\right]= \\
& =\int_{L \cap \partial P}\left[-\eta\left(f_{\infty}\right) \wedge \omega\left(f_{\infty}\right)^{n-d-1}\right]=c_{i} .
\end{aligned}
$$

The last equality comes from [4], Lemma 3, page 51-54.

Therefore $T-R\left(f_{\infty}\right)=0$ in $\Omega-\operatorname{sing}(X)$, that is sunp $\left(T-R\left(f_{\infty}\right)\right) \subset \operatorname{sing}(X)$ and for the support measure theorem (pag. 199) of [5]: $I-R\left(f_{\infty}\right)=0$.

This shows also that every convergent subsequence of $\left\{R\left(f_{v}\right)\right\}$ converges to $R\left(f_{\infty}\right)$ and therefore all the sequence converges to $R\left(f_{\infty}\right)$.

\section{6. - Approximation theorems.}

THEOREM 2. - Let $X$ be a three-dimensional Stein manifold and $\Omega$ a Runge and Stein open of $X, C$ a curve in $\Omega$.

There exists a sequence $\left\{T_{v}\right\} \subset Z_{1}^{+}(X)$ of holomorphic positive 1-cycles such that $\lim _{\nu \rightarrow \infty}\left(\left.T_{v}\right|_{\Omega}\right)=[O]$ in $\mathfrak{D}_{2}(\Omega)$.

PRoof. - Let $\left\{\Omega_{n}\right\}$ be a sequence of relatively compact Stein and Runge in $\Omega$ (and then in $X$ ) opens which invades $\Omega$. 
It is enough to show that for each $n$ there exists $\left\{T_{y m}\right\} \subset Z_{1}^{+}(X)$ such that $\left.T_{p n}\right|_{\Omega_{n}} \rightarrow\left[C \cap \Omega_{n}\right]$.

Let's write $\Omega_{n}=\Omega^{\prime}$, as in Theorem 1 there exist two functions $h_{1}, h_{2}$ holomorphic on $\Omega^{\prime}$ such that:

$$
\left\{x \in \Omega^{\prime}: h(x)=0\right\}=\left(C \cap \Omega^{\prime}\right) \cup \bigcup_{i=1}^{r}\left(Y_{r} \cap \Omega^{\prime}\right)
$$

Therefore $R(h)=c\left[C \cap \Omega^{\prime}\right]+c_{1}\left[Y_{r} \cap \Omega^{\prime}\right]+\ldots+c_{r}\left[Y_{i} \cap \Omega^{\prime}\right]$

But $e=1$; infact let's choose a regular point $x_{0}$ in $O$, because $h_{1}$ and $h_{2}$ generate $\gamma_{0}$ in a neighborhood of $x_{0}$, we can choose a coordinate system at $x_{0}$ such that $h_{1}=z_{1}, h_{2}=z_{2}$ and thus $e=1$ follows from the classical Bochner-Martinelli formula.

Let's put $\mathfrak{J}=\left(\mathfrak{J}_{\mathrm{Y}_{1}}\right)^{c_{1}} \cap \ldots \cap\left(\mathfrak{J}_{\mathrm{Y}_{1}}\right)^{c_{1}}$, since $h \in \Gamma\left(\Omega^{\prime}, \mathfrak{J}\right)$, for the theorem 11 at p. 241 of [3] there exists a sequence $\left\{h_{v}\right\} \subset(\Gamma(X, \jmath))^{2}$ converging to $h$ in $\Omega^{\prime}$.

Thus $R(h)=\left.\lim _{y \rightarrow \infty} R\left(h_{v}\right)\right|_{\Omega^{\prime}}$.

Let's denote by $T_{p} \in Z_{1}^{+}(X)$ the part of $R\left(h_{\nu}\right)$ whose support is not in $Y_{1} \cup \ldots \cup Y_{r}$ except for a zero-dimensional set; we have:

$$
R\left(h_{\nu}\right)=T_{\nu}+c_{1}\left[Y_{1}\right]+\ldots+c_{r^{\nu}}\left[Y_{r}\right]
$$

where $e_{i p} \geqslant c_{i}$ for each $v \geqslant 0$ and $1 \leqslant i \leqslant r$.

Let's choose a point $x_{i} \in\left(\operatorname{reg} Y_{i}\right) \cap \Omega^{\prime}$, let $d_{i}=\operatorname{dim}_{x_{i}}\left(Y_{i}\right)$; let's take at $x_{i}$ a co ordinate set $\left(z_{1}, z_{2}, z_{3}\right)$ and a polycilinder $P$ with eenter $x_{i}$ such that $Y_{i} \cap P=$ $=\left\{x \in P: z_{j}(x)=0\right.$ for each $\left.j \geqslant d_{i}+1\right\}$ and let's denote by $L$ the hortogonal linear space to $Y_{i}$ in $x_{i}$; for the Bochner-Martinelli formula if $v$ is big enough we have:

$$
c_{i}=\int_{L \cap \partial \partial P} \frac{\lambda(h)}{\mid h^{\mid 14}} \quad \text { and } \quad e_{i^{\nu}} \leqslant \int_{L \cap \hat{\partial} P} \frac{\lambda\left(h_{\nu}\right)}{\left|h_{\nu}\right|^{14}}
$$

where $\lambda(h)$ is a form whose coefficients are polynomials in $h$ and its derivatives.

For the continuity of the integration we have $c_{i} \geqslant c_{i p}$ and then $c_{i}=c_{i}$.

Therefore:

$$
R\left(h_{v}\right)=T_{v}+c_{1}\left[Y_{1}\right]+\ldots+c_{r}\left[Y_{r}\right]
$$

and

$$
\left[C \cap \Omega^{\prime}\right]=\lim _{v \rightarrow \infty}\left(\left.T_{v}\right|_{\Omega^{\prime}}\right)
$$

THeorem 3, - Let $X$ be a three-dimensional stein manifold, $\Omega$ a Runge-Stein open of $X$ and $O$ an irriducible curve of $\Omega$.

There exists a sequence $\left\{C_{y}\right\}$ of irriducible curves of $X$ such that

$$
\lim _{p \rightarrow \infty}\left[C_{\eta} \cap \Omega\right]=[C] .
$$

Proof. - It's enough to prove the theorem for each open $\Omega^{\prime}$ relatively compact in $\Omega$. 
Let's fix a relatively compact open $B$ in $\Omega^{\prime}$ containing the singular points of $O \cap \Omega^{\prime}$.

Let now $x$ be a regular point of $C$.

Changing coordinates we can make $C$ a line.

Let fix a polycilinder $U_{x}$ at $x$, if $\left\{T_{v}\right\}$ is a sequence of 1-cyles converging to $O$ as in Theorem 2, for $v$ big enough we have supp $T_{y} \cap U_{x}$ regular and each plane perpendicular to $O$ meets (in $U_{x}$ ) supp $T$ in a simple point by the Bochner-Martinelli formula.

So $\operatorname{supp} T_{v} \cap U_{x}$ is connected with multiplicity one, there exists an irriducible curve $C_{v x}$ of $X$ such that $\left.T\right|_{U_{x}}=\left[C_{v_{x}} \cap U_{x}\right]$ for each $v$ bigger than a suitable $v_{x}$.

It's possible (by instance, triangularizing reg $(C)$ connected) to find a sequence of connected compact invading reg $(C)$.

Then, for $\left(\Omega^{\prime}-B\right) \cap$ reg $C$ is relatively compact in reg $C$, is contained in a connected compact $K$ of 'reg $(C)$.

We can find $x_{1}, \ldots, x_{m} \in K$ such that $U=\bigcup_{i=1}^{m} U_{x_{i}} \supset K$.
Therefore $C \cap \Omega^{\prime} \subset U \cup B$.

And every time $U_{i} \cap U_{j} \neq \emptyset$ we can find $x_{i j} \in U_{i} \cap U_{j}$, a neighborhood $V_{i j} \mathrm{C}$ $\subset U_{i} \cap U_{j}$ of $x_{i j}$ and an integer $\nu_{i j}$ such that for $\nu \geqslant \nu_{i j}\left(\operatorname{supp} T_{p}\right) \cap V_{i j}$ is not empy and irreducible.

Since $U$ is connected ( $K$ is connected) for $v \geqslant \max \left\{v_{i}, v_{i j}\right\}$ the irriducible curve representing $T_{v}$ in each $U_{x_{i}}$ must be the same, then

$$
\left.T_{v}\right|_{U}=\left[C_{v} \cap U\right]
$$

For $v$ big enough, we have $\left(\operatorname{supp} T_{v}\right) \cap \Omega^{\prime} \subset(U \cup B) \cap \Omega^{\prime}$.

To prove it, a way is to remember (Theorem 2) that the supp $T_{v}$ are zero-sets of holomorphic maps going to $f$ whith zero-set $C$.

Therefore $\left.\left(T_{v}-\left[C_{v}\right]\right)\right|_{U}=0$, then supp $\left(T_{v}-\left[C_{v}\right]\right) \subset B$, that is

$$
\operatorname{dim}\left(\operatorname{supp}\left[\left.\left(T_{v}-\left[C_{v}\right]\right)\right|_{\Omega^{\prime}}\right]\right)=0
$$

and the measure support theorem (page 199 of [5]) shows $T_{v}=\left[C_{v}\right]$ on $\Omega^{\prime}$.

\section{REFERENCES}

[1] A. ANDREOTTI - W. STOLL, Analytic and algebraic dependence of meromorphic functions, Lecture notes 234, Springer-Verlag (1971).

[2] O. Fonstre - K. J. RAMspot, Analytische Modulgarben und Endromisbündel, Inv. Math., 2 (1966), pp. 145-170.

[3] R. C. Gunnixg - H. Rossi, Analytic funotions of several complex variables, Prentice Hall series (1965).

[4] J. R. KnNa, A residue formula for complex subvarieties, Carolina conference proceedings (1970), pp. 43-56.

[5] J. R. KNNG, The currents defined by analytic varieties, Aeta Math., 127 (1971), pp. 185-220.

[6] R. Narashiman, On the homology groups of Stein spaces, Inv. Math., 2 (1967), pp. 377-385.

[7] N. STEenrod, The topology of fibre bundles, Princeton Mathematical series, No. 14 (1951). 